\title{
DETERMINATION OF COMPLETE O.D.F.S UNDER ASSUMPTION OF A MINIMAL VALUE
}

\author{
F. WAGNER, M. HUMBERT, C. ESLING and J. MULLER \\ LM2P, Faculté des Sciences, Ile du Saulcy, \\ 57045 Metz-Cedex 01, France
}

\section{Introduction}

In the harmonic method the calculation of a complete O.D.F. from pole figure data is worked out in two distinct stages:

- the first one aims to calculate the reduced O.D.F., $\tilde{f}(\mathrm{~g})$, (also called the even part of the O.D.F.)which makes it possible to recalculate any pole-figure. Because of experimental errors of the pole figure data, the reduced O.D.F. may correspond to recalculated pole-figures which exhibit some negative values. Dahms and Bunge have proposed to use an iterative technique which intends to ensure a physical meaning to the reduced O.D.F. i.e. recalculated pole-figures which are positive everywhere $/ 1 /$. The interest of this technique in the harmonic method will be illustrated at the end of the present paper.

-the second stage of the texture analysis consists in calculating a complete O.D.F., $\mathrm{f}(\mathrm{g})$, starting from the reduced one, $\tilde{f}(g)$ (this means that an odd part, $\widetilde{f}(g)$, which has no influence on pole-figures, is added to the even one). Several possibilities have been suggested for this purpose : the zero range method $/ 2 /$, the gaussian peaks $/ 3 /$, the quadratic method /4/. More recently Dahms and Bunge have proposed to use an iterative technique once more to calculate a complete O.D.F. by using the positivity condition $f(g) \geq 0 \quad / 5 /$. They have also emphasized that a condition $\mathrm{f}(\mathrm{g}) \geq \mathrm{rmin}$ can be used, instead of the positivity condition, which means that a solution is searched with a given isotropic component or "phon", rmin (obvioulsy $0 \leq \mathrm{rmin} \leq 1$ ). The present paper tries to answer the question : how to choose such a rmin value and what the effect of this choice is on the determination of the complete O.D.F. 


\section{Determination of a complete O.D.F. by the iterative technique}

Assuming that a reduced O.D.F., $\tilde{\mathbf{f}}(\mathrm{g})$, has been calculated from the experimental pole figures the complete O.D.F. can thus be determined in an iterative way and reads at the $n^{\text {th }}$ step

$$
f_{n}(g)=f_{n-1}(g)+\lambda_{n} \widetilde{\widetilde{f}}_{n}(g)
$$

$$
=0 \quad \text { if } \mathrm{f}_{\mathrm{n}-1}(\mathrm{~g}) \geq \mathrm{r}_{\min }
$$

with $\hat{\mathbf{f}}_{\mathrm{n}}(\mathrm{g})\{$

and

$$
f_{0}(g)=\tilde{f}(g)
$$

and where $\lambda_{n}$ is an optimization parameter which minimizes the distance of the current solution, $f_{n}(g)$, to the set of admissible solutions satisfying $f(g) \geq r$ m in $/ 6,7 /$.

The symbol $\approx$ appearing over $\hat{f}(g)$ in relation (1) means that only the odd part of this function is added so that in the final solution the even part, $\tilde{f}(g)$ is never modified along the iterations.

\section{Results for a theoretical example.}

To test the effect of the choice of rmin on the calculated O.D.F. we have first used the so-called "Santa-Fe example" proposed by Matthies /8/. In this example the O.D.F. runs from $f_{\min }^{\text {true }}=0.72$ up to $f_{\max }^{\operatorname{true}}=5.04$ whereas the reduced O.D.F. is between $\quad \tilde{\mathbf{f}}_{\min }^{\text {true }}=0.07$ and $\tilde{\mathbf{f}}_{\max }^{\text {true }}=3.96$.

Starting from the corresponding pole-figures we have first determined $\tilde{f}(g)$ and then calculated $f(g)$ for 13 different 
situations i.e. for 13 different rmin values. The results are not discussed in terms of O.D.F. sections because most of them are very similar but only in terms of maximum and minimum. At this point it is important to distinguish between $\mathrm{rmin}$ which is the requested minimum introduced in the iterative technique and fmin which is the minimal value of the calculated O.D.F.

When starting from $\tilde{f}(g)$, if it exists a solution $f(g)$ with $\mathrm{fmin}=$ rmin the algorithm will of course find it; if such a solution does not exist the calculated O.D.F. will have a minimal value fmin $\neq \mathrm{rmin}$.

Figure 1 shows both the maximum, fmax, and minimum, fmin, of each calculated O.D.F.. It appears clearly that as long as rmin is less than fmin the calculated O.D.F. is somewhat different from the true O.D.F.. On the contrary if $\mathrm{rmin}$ is greater than $\mathbf{f}_{\mathrm{min}}^{\mathrm{true}}$ the calculated O.D.F. is very close to the true one. This is important because in a texture analysis the available information is the minimal value in the pole-figures Pmin $=\left\{\right.$ Min $P_{h i}(y), \forall$ hi,$\left.\forall y\right\}$ which is known to obey the inequality :

$$
\text { fmin } \leq \mathrm{P} \text { min }
$$

Then by setting $\mathrm{rmin}=$ Pmin in the O.D.F. calculation a solution will be found with the maximal phon and the fact that rmin could be an overestimation of the isotropic component, true

$f_{\mathrm{min}}$, has a small influence on the quality of the result.

In this Santa Fe example Pmin $=0.72$; by using $\mathrm{rmin}=P \min$ $=0.72$ in the texture analysis it can be seen from Figure 1 that the calculated O.D.F. is very close to the true O.D.F.. 


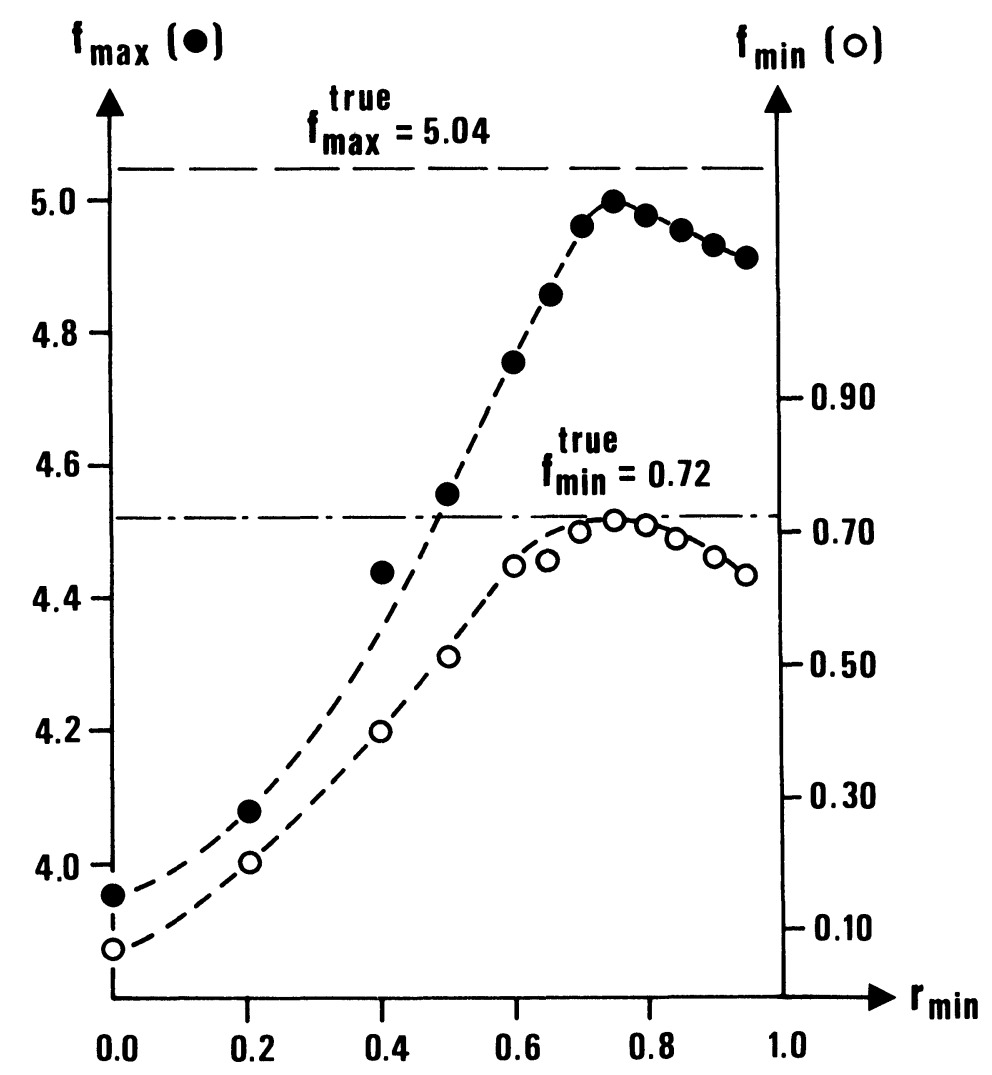

Figure 1 : Maximal and minimal value of the O.D.F., fmax (•) and fmin (o), as a function of rmin for the Santa-Fe example

\section{Results for a real specimen}

Three incomplete pole-figures have been measured in a "standard" way (up to $\phi_{\mathrm{max}}=75^{\circ}$, steps of $5^{\circ}$ in azimuth and declination ) and 5 texture analyses have been performed 
from these data. In the first texture analysis (TA number 1) no positivy is activated during the first stage of analysis and the complete O.D.F. is calculated with $\mathrm{rmin}=0$.

On the contrary, for the four other analyses, the reduced O.D.F. is determined with positivy technique before to perform the second stage of calculation for the complete O.D.F. with rmin $=0 ., 0.2,0.4$ and 0.6 respectively.

In table $I$ are reported the minimal and maximal values, fmin and fmax, of the calculated O.D.F., $f(g)$, and figure 2 shows some sections of the O.D.F. corresponding to the texture analyses numbered TA1, TA2 and TA5.

\begin{tabular}{|l|l|l|l|}
\hline $\begin{array}{l}\text { Texture } \\
\text { Analysis } \\
\text { number }\end{array}$ & $\begin{array}{l}\text { requested } \\
\text { minimum } \\
\text { rmin }\end{array}$ & $\begin{array}{l}\text { minimal } \\
\text { value of } \\
\mathrm{f}(\mathrm{g}): \text { fmin }\end{array}$ & $\begin{array}{l}\text { maximal } \\
\text { value of } \\
\mathrm{f}(\mathrm{g}): \mathrm{fmax}\end{array}$ \\
\hline TA 1 & 0. & -2.30 & 10.41 \\
\hline TA 2 & 0. & -0.13 & 9.51 \\
\hline TA 3 & 0.2 & -0.23 & 9.51 \\
\hline TA 4 & 0.4 & -0.26 & 9.57 \\
\hline TA 5 & 0.6 & -0.30 & 9.54 \\
\hline
\end{tabular}

Table I : Calculated minimal and maximal values of $f(g)$, fmin and fmax, for 5 different texture analyses from the same set of 3 incomplete pole-figures of a low carbon steel.

It appears clearly that without positivity at the first stage (TA number 1) no satisfactory solution is found (the minimal value, fmin, is greatly negative). For the other four analyses realistic results are obtained with fmin near zero (fmin decreases very slowly with increasing $\mathrm{rmin}$ ). 
F. WAGNER $E T A L$

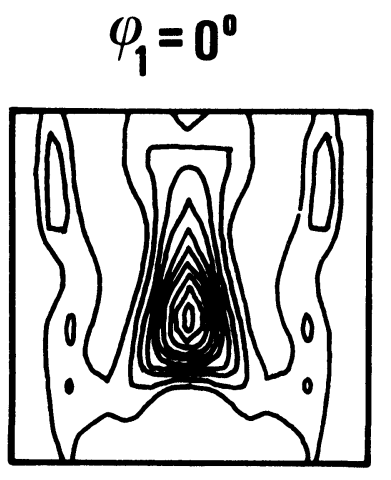

$\varphi_{1}=45^{\circ}$

$$
\varphi_{1}=90^{\circ}
$$
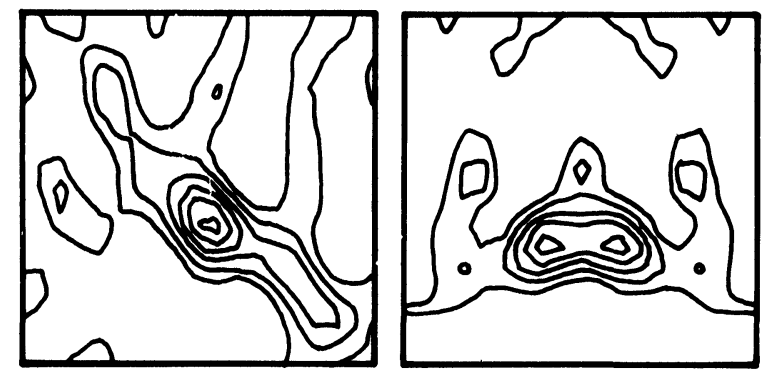

a)
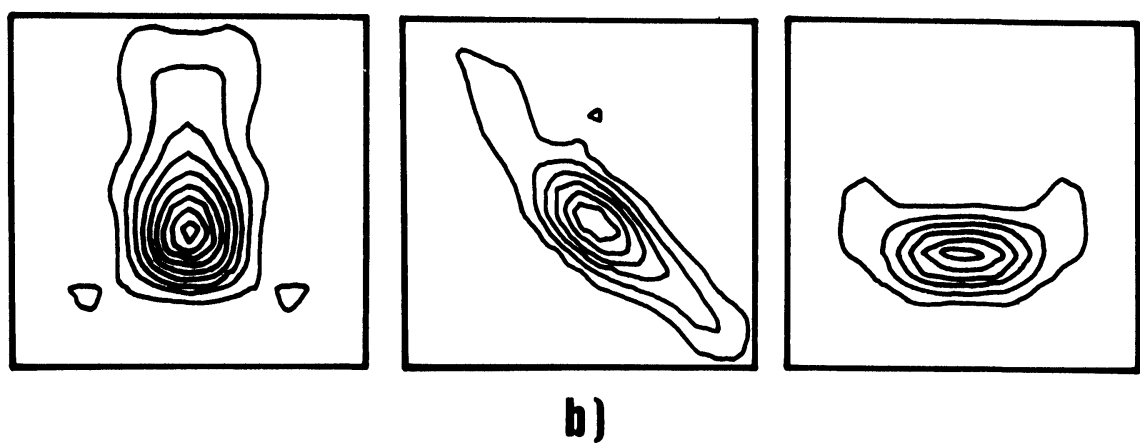

b)
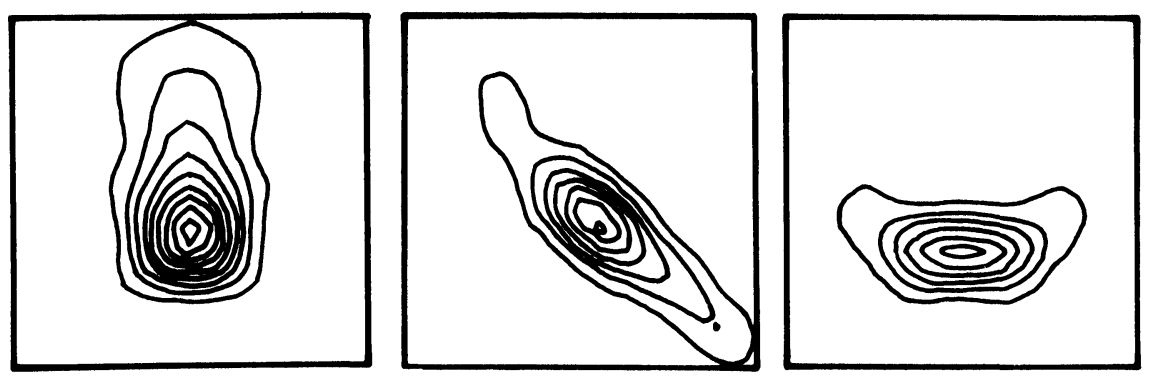

C)

Figure 2 : Three O.D.F. sections $\left(\varphi_{1}=0^{\circ}, \varphi_{1}=45^{\circ}, \varphi_{1}=90^{\circ}\right)$ of an annealed low carbon steel for several analysis conditions : a) TA 1 ; b) TA 2 ; c) TA 5

levels : 1 . ,2. 3. , 4. 5. ,6. , 7. ,8. ,9. 


\section{Conclusion}

The previous results allow to emphasize the two following points :

- a "bad" solution $\tilde{f}(g)$ at the first stage of the analysis prevents a further satisfactory result for the complete O.D.F., $\mathrm{f}(\mathrm{g})$, as it was illustrated in the case of a steel. This obvious statement means that the iterative technique of positivy is necessary at both stages of the texture analysis with the harmonic method.

- by choosing rmin $=$ Pmin for the calculation of the complete O.D.F., a solution is reached which is very closed to the one obeying the requirement of maximal phon without the necessity of iterations on this phon as in the WIMV method /8/. This last point has likely to be restricted to the case of texture functions with plateau (i.e. functions which present a minimal value in a large part of the Euler space as in the two studied examples). This is fortunately the most usual case in texture analysis.

\section{References}

/ 1 / M.Dahms and H.J. Bunge, J. Appl. Cryst., 22, p. 439,(1989)

/ 2 / H.J.Bunge and C.Esling, J. Phys. Let.,40.p.L -627, (1979)

/ 3 / K.Lücke, J.Pospiech, K.H. Virnich and J.Jura, Acta Met, 29. p.167, (1981)

/ 4 / P.Van Houtte, Textures and Microstuctures, 6, p.1, (1980)

/ 5 / M.Dahms and H.J. Bunge, Textures and Microstructures, 10 p.21, (1988)

/ 6 / F.Wagner, M. Humbert, J. Muller and C. Esling, Europhys. Lett., 11, p. 479, (1990).

/ 7 / F.Wagner and M.Dahms, in Advances and applications of Quantitative Texture Analysis, ed. H.J. Bunge and C. Esling, DGM Oberursel, in print.

/ 8 / S.Matthies, Proceedings of ICOTOM 8, ed. J S. Kallend and G. Gottstein, p.37, (1988) 